\title{
THE CENTER AND CYCLICITY PROBLEMS FOR QUARTIC LINEAR-LIKE REVERSIBLE SYSTEMS
}

\author{
LEONARDO P. C. DA CRUZ, VALERY G. ROMANOVSKI, AND J. TORREGROSA
}

\begin{abstract}
In this paper we study a family of quartic linear-like reversible polynomial systems having a nondegenerate center at the origin. This family has degree one with respect to one of the variables. We are interested in systems in this class having two extra nondegenerate centers outside the straight line of symmetry. The geometrical configuration of these centers is aligned or triangular. We solve the center problem in both situations and, in the second case, we study the limit cycles obtained from a simultaneous degenerate Hopf bifurcation in the quartic polynomials class.
\end{abstract}

\section{INTRODUCTION}

Let us consider a planar analytic system of ordinary differential equations defined in a neighborhood of the origin, $(\dot{x}, \dot{y})=(f(x, y), g(x, y))$. We are interested in the local structure of the solutions near an equilibrium point of nondegenerate center-focus type located at the origin. That is, a point with the Jacobian matrix having nonzero determinant and null trace. More specifically, assuming $f(0)=g(0)=0$, if we write the eigenvalues of the Jacobian matrix of $(f, g)$ at 0 as $\alpha \pm i \beta$, then $\alpha=0$ and $\beta \neq 0$. So, after a time rescaling, we can assume that $\beta=1$, and we can write the corresponding system in the canonical form

$$
(\dot{x}, \dot{y})=\left(-y+f_{n}(x, y), x+g_{n}(x, y)\right)
$$

where $f_{n}$ and $g_{n}$ are polynomials of degree $n$, which do not contain neither constant nor linear terms. The problem of distinguishing whether the singular point at the origin of (1) is a center or a focus is known as the Poincaré center problem, the center-focus problem, or just the center problem. Even this problem was partially solved by Lyapunov, see [20], it has been studied for some fixed values of the degree $n$ during more than a century by many authors. The only family completely investigated is the quadratic one. The study of this family was started by Dulac in 1908 in [11], and also performed by Kapteyn some years later, see $[18,19]$. Up to the work of Frommer ([14]), the coefficient center conditions for existence of a center were not published. The problem was investigated for the quadratic system in the real form and the computations were rather difficult. The correct center conditions were published by Saharnikov ([28]) and later by Sibirskiı $([31,32])$. The center conditions are simpler and the center-focus problem is easier to solve if the system is written in complex coordinates, see [35]. For the complete cubic family (when in (1) $n=3$ ), the problem remains unsolved. Only particular families has been studied, for example the linear plus cubic homogeneous in [33] or cubic with degenerate infinity in [3]. Some recent works with other cubic families are [1, 7, 8, 29, 30]. For higher degree systems, none of the homogeneous nonlinearity cases $n=4$ and $n=5$ are completely solved, see [2, 13]. In last thirty years, there is a long list list of published papers studying many particular polynomial families (see, for example, the references in $[13,25])$.

2010 Mathematics Subject Classification. Primary 34C07, 34C23, 37C27.

Key words and phrases. Center-focus problem, linear-like reversible centers, quartic centers, center cyclicity. 
In this work we are interested in the limit cycles bifurcating simultaneously from reversible centers with two extra symmetric centers out of the symmetry line. All considered centers are nondegenerate. The aim is to do a similar study as the one done by Christopher in [6] or by Prohens and Torregrosa in [24]. In this last work, the classification problem for cubic systems having such properties was done. The natural continuation problem is the study of the cyclicity of reversible quartic systems having a center at the origin. These systems can be written as

$$
\begin{aligned}
& \dot{x}=-y+a_{11} x y+a_{21} x^{2} y+a_{03} y^{3}+a_{31} x^{3} y+a_{13} x y^{3}, \\
& \dot{y}=x+a_{20} x^{2}+a_{02} y^{2}+a_{30} x^{3}+a_{12} x y^{2}+a_{40} x^{4}+a_{22} x^{2} y^{2}+a_{04} y^{4} .
\end{aligned}
$$

Before starting with the simultaneous bifurcation it is necessary to solve the center-focus problem for (2). This classification problem has been impossible to finish, as similar problems having too many parameters. We remark that the above system has 12 parameters. In order to simplify and convert this problem to some tractable one, we propose a restricted family following the ideas in [22]. We consider the quartic linear-like reversible systems, that is, all polynomial systems with degree four, time reversible with respect to the $x$-axis, and having degree one also in the variable $x$. This family of systems writes as

$$
\begin{aligned}
& \dot{x}=-y+a_{11} x y+a_{03} y^{3}+a_{13} x y^{3}, \\
& \dot{y}=x+a_{02} y^{2}+a_{12} x y^{2}+a_{04} y^{4} .
\end{aligned}
$$

We recall that the above family is invariant with respect to the change of variables $(x, y, t) \rightarrow(x,-y,-t)$.

In Section 3 we will prove that system (3), having two extra symmetric centers out of the symmetry line, can be written in two different canonical forms. The first corresponds to the case with three aligned centers over $x=0$ and the second with three centers in a triangular position. The following two results provide the answer to the center-focus classification problem.

Theorem 1.1. The reversible quartic system (3) having three aligned singularities of center-focus type can be written as

$$
\begin{aligned}
& \dot{x}=-y-(2 c+b) x y+y^{3}+b y^{3} x, \\
& \dot{y}=x-c y^{2}-\frac{1}{2}\left(a^{2}+4 c^{2}+2\right) x y^{2}+c y^{4},
\end{aligned}
$$

with $a>0$ and $c \geq 0$ (see Figure 1 right). Moreover, the above system has three aligned centers at $(0,0)$ and $(0, \pm 1)$ if and only if one of the following conditions holds:

$\left(\mathcal{A}_{1}\right) c=0$

$\left(\mathcal{A}_{2}\right) b=a^{2}-4 c^{2}+2=0$.
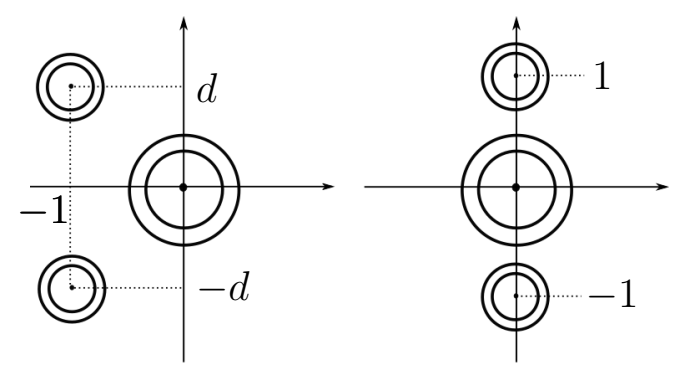

FiguRE 1. The two different configurations of centers of system (3) 
Theorem 1.2. The reversible quartic system (3) having three singularities of center-focus type in a triangular position can be written as

$$
\begin{aligned}
& \dot{x}=-y-\frac{(c+d) x y}{d}+\frac{(b+d) y^{3}}{d^{3}}+\frac{(c+b+d) x y^{3}}{d^{3}} \\
& \dot{y}=x+\frac{\left(a^{2} d+b^{2} d+b c d+2 c\right) y^{2}}{2 c d^{2}}+\frac{\left(a^{2} d+b^{2} d-2 c\right) x y^{2}}{2 c d^{2}}-\frac{(b d+2) y^{4}}{2 d^{4}},
\end{aligned}
$$

with $a>0, c \neq 0$, and $d>0$. See Figure 1 left. Moreover, the above system has three centers, one at the origin and two more at $(-1, \pm d)$, if and only if one of the following conditions holds:

$\left(\mathcal{T}_{1}\right) a^{2} d-2 c=b=0 ;$

$\left(\mathcal{T}_{2}\right) b d+2=a^{4}+a^{2} b^{2}-a^{2} c^{2}-2 a^{2} c d-a^{2} d^{2}+4 a^{2}+4 b^{2}+4 b c-4=0 ;$

$\left(\mathcal{T}_{3}\right) d^{2}-b d+c d-4=2\left(a^{2}+b^{2}\right)+b c-c^{2}-c d=0 ;$

$\left(\mathcal{T}_{4}\right) d-2=c+1=a^{2}+b^{2}-b=0 ;$

$\left(\mathcal{T}_{5}\right) c d+4=b+c+d=a^{2}+d^{2}-4=0 ;$

$\left(\mathcal{T}_{6}\right) c d+d^{2}+2=b+c+d=a^{2}-2 c^{2}+2 d^{2}+10=0 ;$

$\left(\mathcal{T}_{7}\right) c d+d^{2}-2=a^{2}+b^{2}+b c=0$

$\left(\mathcal{T}_{8}\right) d-2=b-c=a^{2}+c^{2}-c=0$;

$\left(\mathcal{T}_{9}\right) 2 c+3 d=2 b-d=d^{2}-4 a^{2}-4=0$;

$\left(\mathcal{T}_{10}\right) b+c+d=a^{2} d-c^{2} d-2 c d^{2}-d^{3}+2 c+4 d=0 ;$

$\left(\mathcal{T}_{11}\right) c d+d^{2}-2=2 b-d=d^{2}-4 a^{2}-4=0 ;$

$\left(\mathcal{T}_{12}\right) c d+d^{2}-6=3 b-c-d=9 a^{2}-2 c^{2}+2 d^{2}-6=0$

$\left(\mathcal{T}_{13}\right) c d+d^{2}-2=c^{2} d-b d^{2}-d^{3}+2 b+4 d=b c+c^{2}-d^{2}+4=a^{2}+b^{2}-c^{2}+d^{2}-4=0 ;$

$\left(\mathcal{T}_{14}\right) c d-2=b+c-d=a^{2}+d^{2}-4=0 ;$

$\left(\mathcal{T}_{15}\right) c^{2} d^{2}+2 c d^{3}+d^{4}-c d-2 d^{2}-2=b+c+d=c^{3} d-3 c d^{3}-2 d^{4}+a^{2}-2 c^{2}-2 c d+3 d^{2}+8=0 ;$

$\left(\mathcal{T}_{16}\right) c d-2=b=a-c=0$

$\left(\mathcal{T}_{17}\right) c d+2 d^{2}-8=2 b-c=a^{2}+d^{2}-4=0 ;$

$\left(\mathcal{T}_{18}\right) a^{2}+c d+d^{2}=b+c+d=0$.

The paper is structured as follows. In Section 2 we present some preliminary results on the center conditions computation, the Darboux integrability, and the degenerate Hopf bifurcation problem. Section 3 is devoted to deriving the canonical forms (4) and (5). The center classification is done in Section 4. Finally, the simultaneous limit cycles bifurcation in some systems with three centers listed above are studied in Section 5.

\section{Preliminaries}

In this section we recall some classical concepts, necessary to state and prove the results of this paper. In Section 2.1 we provide the definition and computational algorithm to compute the center conditions which we use to study the center-focus problem. In Section 2.2 we explain how the simultaneous degenerate Hopf bifurcations are done for proving the results of Section 5. Finally, some results on the Darboux integrability are given in Section 2.3. 
2.1. The center conditions. The approach to characterize when system (1) has a center at the origin is based on the well known Poincaré-Lyapunov Theorem, see [21, 23]. Before stating it, we recall the definition of first integral. We say that a nonconstant analytical function defined in a neighborhood $\Omega$ of the origin, $\Phi: \Omega \subset \mathbb{R}^{2} \rightarrow \mathbb{R}^{2}$, is a first integral of system (1) if it is constant along any solution $\gamma$ or, equivalently,

$$
\dot{x} \frac{\partial \Phi}{\partial x}+\left.\dot{y} \frac{\partial \Phi}{\partial y}\right|_{\gamma} \equiv 0 .
$$

Theorem 2.1. System (1) has a center at the origin if and only if it admits a local analytic first integral of the form

$$
\Phi(x, y)=x^{2}+y^{2}+\sum_{k+\ell \geq 3} q_{k, \ell} x^{k} y^{\ell} .
$$

Moreover, the existence of a formal first integral $\Phi$ of the above form implies the existence of a local analytic first integral of the same form.

For a proof of this result we refer to [17, 25].

The necessary conditions for the existence of a first integral (7) for system (1) are obtained looking for a formal series (7) satisfying (6). To start the computational procedure for finding the first $N$ conditions for integrability, we write down (7) up to order $2 N+2$

$$
\widetilde{\Phi}(x, y)=x^{2}+y^{2}+\sum_{k+\ell \geq 3}^{2 N+2} q_{k, \ell} x^{k} y^{\ell} .
$$

Then, for each $i=3, \ldots, 2 N+2$ we equate to zero the coefficients of terms of degree $i$ in the expression

$$
\dot{x} \frac{\partial \widetilde{\Phi}}{\partial x}+\dot{y} \frac{\partial \widetilde{\Phi}}{\partial y}=\left(-y+f_{n}(x, y)\right) \frac{\partial \widetilde{\Phi}}{\partial x}+\left(-x+g_{n}(x, y)\right) \frac{\partial \widetilde{\Phi}}{\partial y} .
$$

Starting at $i=3$, we should solve in a recurrence way each linear system of $i+1$ equations with $i+1$ variables, $q_{k, \ell}$ such that $k+\ell=i$. All linear systems corresponding to odd degrees, $i=2 j+1$, have a unique solution in terms of previous values of $q_{k, \ell}$. As the determinant of the linear system that corresponds to an even degree, $i=2 j+2$, vanishes, we need to add an extra condition in order that the linear system has a unique solution. In fact, at this step, we have one equation more than the number of variables. We add suitable equations, for the terms $x^{2 j+2}$ for example, in order that the derivative over the associated vector field writes as

$$
\dot{x} \frac{\partial \Phi}{\partial x}+\dot{y} \frac{\partial \Phi}{\partial y}=\sum_{j=1}^{\infty} L_{j} x^{2 j+2} .
$$

Then, when at least one $L_{j}$ is different from zero $\Phi$ it is a Lyapunov function in a neighborhood of the origin. Then, system (1) has no local analytic first integral and we say that the equilibrium point is a weak focus of order $k$ if the first nonzero coefficient in (9) is $L_{k}$. The coefficient $L_{j}$ in (9) is called the $j$-th Lyapunov quantity. The stability of the origin is given by the sign of the first nonzero $L_{j}$.

It is obvious that $L_{j}$ are polynomials in the parameters of system (1). Clearly, for $N$ big enough, the above algorithm provides a necessary set of conditions, $\left\{L_{j}=0: j=\right.$ $1, \ldots, N\}$, for system (1) be a center. In other words, we can also say that the polynomials $L_{j}$ represent obstacles for the existence of a first integral. In particular, system (1) admits a first integral of the form (7) if and only if $L_{j}=0$, for all $j \geq 1$. Thus, the simultaneous 
vanishing of all focus quantities provides conditions which characterize when a system of the form (1) has a center at the origin.

The next definition recalls the notion of Bautin ideal and the center variety.

Definition 2.2. The ideal defined by the real focus quantities, $\mathcal{B}^{\mathbb{R}}=\left\langle L_{1}, L_{2}, \ldots\right\rangle \subset \mathbb{C}[\lambda]$, where $\lambda$ represents all the parameters of system (1), is called the real Bautin ideal. The affine variety $\mathbf{V}^{\mathbb{R}}=\mathbf{V}\left(\mathcal{B}^{\mathbb{R}}\right)$ is called the real center variety of system (1).

By the Hilbert Basis Theorem there exists a positive integer $j$ such that $\mathcal{B}^{\mathbb{R}}=\mathcal{B}_{j}^{\mathbb{R}}=$ $\left\langle L_{1}, \ldots, L_{j}\right\rangle$. The main difficulty is that there is no technique to get $j$ a priori. Notice that the inclusion $\mathbf{V}^{\mathbb{R}}=\mathbf{V}\left(\mathcal{B}^{\mathbb{R}}\right) \subset \mathbf{V}\left(\mathcal{B}_{j}^{\mathbb{R}}\right)$ holds for any $j \geq 1$. The opposite inclusion, for a fixed $j$, is verified finding the irreducible decomposition of $\mathbf{V}\left(\mathcal{B}_{j}^{\mathbb{R}}\right)$, see [25], such that any point of each component of the decomposition corresponds to a system having a center at the origin. To find the irreducible decomposition of $\mathbf{V}\left(\mathcal{B}_{j}^{\mathbb{R}}\right)$, we perform the computations with the Computer Algebra System SINGULAR $([9,10])$. In Section 4 we explain this procedure in more detail.

2.2. Degenerate Hopf bifurcation. Roughly speaking, we can say that the cyclicity of an equilibrium point is the maximum number of isolated periodic orbits bifurcating from it. We are interested only in the limit cycles bifurcating from nondegenerate monodromic points, where the return map is well defined and analytic. Moreover, both unperturbed systems and perturbations are polynomials of degree four in the variables $x, y$.

With the notation introduced above, the classical bifurcation known as the Hopf bifurcation is the emergence of a limit cycle from a weak-focus of first order varying the trace of the matrix of the linearized system from zero to a small enough but nonzero value. We will denote by $L_{0}$ the trace of the perturbed system, clearly $L_{0}=0$ for the unperturbed one. More concretely, the origin of the unperturbed system is stable (resp. unstable) when $L_{1}<0$ (resp. $L_{1}>0$ ). Then, the perturbed system, when $L_{0}$ is a small enough positive (resp. negative) real number, a small stable (resp. unstable) limit cycle bifurcates from the origin. This is because the monodromic property remains but the local stability of the equilibrium point changes from stable to unstable. The degenerate Hopf bifurcation is the natural generalization of this bifurcation phenomenon when $k$ small limit cycles appear from a weak-focus of order $k$.

In general, the complete unfolding of $k$ limit cycles near a weak focus of order $k$ is only guarantied when the perturbation is analytic, see for example [27]. When the perturbation is restricted to be polynomial of some fixed degree this property is not automatic. This is the case in our problem. Our unperturbed systems (3) are of degree four and we are perturbing them in the full quartic polynomial systems. This is the main reason why the problem of finding the cyclicity of a center, for systems like (3), is so difficult. A way to avoid this difficulties is to study lower bounds for the cyclicity. This is the aim of the next result due to Christopher ([6]) that, as a direct application of the Implicit Function Theorem, provides necessary conditions to get lower bounds for the cyclicity of a center. In fact, there are similar previous results due to Chicone and Jacobs ([4, 5]). Also Han ([16]) applies them for Liénard families.

Theorem 2.3. Suppose that $\boldsymbol{c}$ is a point on the center variety in the parameter space and that the first $L_{1}, \ldots, L_{k}$ Lyapunov quantities have independent linear parts (with respect to the expansion of them with respect all perturbation parameters), then $\boldsymbol{c}$ lies on a component of the center variety of codimension at least $k+1$, adding the trace parameter $L_{0}$, and there are bifurcations which produce $k$ limit cycles locally from the center corresponding to the parameter value $\boldsymbol{c}$. 
In particular, the above result shows the existence of a curve of weak-foci of order $k$ that unfold $k$ hyperbolic limit cycles. The existence of this curve is obtained studying the Taylor developments of the varieties $L_{j}=0$, for $j=0, \ldots, k$, that intersect transversally along it but $L_{k}$ is nonvanishing.

Finally, in [6] there are results about the existence of such transversal curves studying the homogeneous higher order terms when the previous ones vanish identically. They will be used in the future work to improve the results of this paper.

In this work we are interested in the simultaneous bifurcation of limit cycles in the three center configuration that a system of type (3) has. We will explain how we use Theorem 2.3 for this purpose in Section 5 .

2.3. Darboux integrability. More detailed versions of the next results can be found in $[12,25]$. Here we only remind the statement on Darboux integrability that we will need for proving our classification results, together with some basic concepts and definitions.

Let $(\dot{x}, \dot{y})=(P(x, y), Q(x, y))$ be a $\mathbb{C}$-polynomial differential system of degree $m$. We say that an algebraic curve $f=0$ is invariant if the vector field associated to the polynomial differential system is tangent along it. That is,

$$
P(x, y) \frac{\partial f}{\partial x}+Q(x, y) \frac{\partial f}{\partial y}=K(x, y) f(x, y) .
$$

The polynomial $K$ is known as the corresponding cofactor. Equivalently, $\exp (g / h)$ is called an exponential factor, with the associated cofactor $K^{\mathrm{e}}$, if it satisfies

$$
P(x, y) \frac{\partial \mathrm{e}^{g / h}}{\partial x}+Q(x, y) \frac{\partial \mathrm{e}^{g / h}}{\partial y}=K^{\mathrm{e}}(x, y) \mathrm{e}^{g / h} .
$$

Theorem 2.4. Suppose that a $\mathbb{C}$-polynomial differential system of degree $m$ admits $p$ irreducible invariant algebraic curves $f_{i}=0$ with cofactors $K_{i}$ for $i=1, \ldots, p$ and $q$ exponential factors $\exp \left(g_{j} / h_{j}\right)$ with cofactors $K_{j}^{\mathrm{e}}$ for $j=1, \ldots, q$. Then, there exist complex numbers $\lambda_{i}$ and $\mu_{j}$ not all zero such that $\sum_{i=1}^{p} \lambda_{i} K_{i}+\sum_{j=1}^{q} \mu_{j} K_{j}^{\mathrm{e}}=0$, if and only if the (multivalued) function

$$
f_{1}^{\lambda_{1}} \cdots f_{p}^{\lambda_{p}}\left(\exp \left(\frac{g_{1}}{h_{1}}\right)\right)^{\mu_{1}} \cdots\left(\exp \left(\frac{g_{q}}{h_{q}}\right)\right)^{\mu_{q}}
$$

is a first integral of the $\mathbb{C}$-polynomial differential system of degree $m$.

\section{CANONICAL FORM}

As we have already mentioned, system (3) is time-reversible with respect to the $x$-axis, and it has a nondegenerate equilibrium point of center-focus type at the origin. Clearly, the reversibility condition and the linear part ensures that the origin will be always a center. As we have explained in the introduction, the aim of this paper is the study of system (3) having two extra nondegenerate centers out of the symmetry line. Then, as they can be located at $\left(x_{0}, \pm y_{0}\right)$, there are two different possibilities, $x_{0}=0$ and $x_{0} \neq 0$. In the rest of the section we will prove the first part of the statements of Theorems 1.1 and 1.2 .

In the first case, $x_{0}=0$, we can locate the equilibrium points at $(0, \pm 1)$ after a rescaling. Then, imposing that the determinants of the Jacobian matrix at these points writes as a positive number, $a^{2}$, we get system (4). We notice that by the symmetry both determinants coincide. We can also assume the condition $c \geq 0$ doing, if necessary, the 
change $(x, y) \rightarrow(-x,-y)$. Hence, with the new variables $(u, v)=(x,-1+y)$, there exists a first integral that writes

$$
H(u, v)=(a u)^{2}+4(c u-v)^{2}+\cdots .
$$

In the second case, $x_{0} \neq 0$, we can locate the equilibrium points at $(-1, \pm d)$ after a rescaling, if necessary. The symmetry provides the condition $d>0$. Assuming that the system has an equilibrium point with zero trace we get

$$
\begin{aligned}
& a_{02}=\frac{d^{4} a_{13}+d^{2}\left(a_{11}+2 a_{12}\right)+4}{2 d^{2}}, \\
& a_{03}=\frac{d^{2} a_{13}+a_{11}+1}{d^{2}}, \\
& a_{04}=-\frac{d^{4} a_{13}+d^{2} a_{11}+2}{2 d^{4}} .
\end{aligned}
$$

Then, adding the condition that it has a positive determinant, $a^{2}$, we obtain

$$
a_{12}=-\frac{d^{6} a_{13}^{2}+2 d^{4} a_{11} a_{13}+d^{2} a_{11}^{2}+a^{2}+2 a_{11}+2}{2 d^{2}\left(a_{11}+1\right)} .
$$

Clearly, it is not restrictive if we assume $a>0$. The above denominator never vanishes because, when $a_{11}=-1$, the determinant, $a^{2}=-d^{2}\left(a_{13} d^{2}-1\right)^{2}$, would be negative. Finally, we introduce two new parameters $(b, c)$ performing the linear change

$$
a_{11}=-\frac{c+d}{d}, \quad a_{13}=\frac{c+b+d}{d^{3}} .
$$

Hence, with the new variables $(u, v)=(1+x,-d+y)$, there exists a first integral that writes

$$
H(u, v)=(a d u)^{2}+(-b d u+2 c v)^{2}+\cdots .
$$

This expression can be easily transformed to (7). We remark that the condition $a_{11}+1 \neq 0$ is equivalent to $c \neq 0$. From all the above transformations the system (3) changes to (5).

We notice that, although the last parameter change does not seem necessary, it will help to obtain simpler expressions for the center conditions in the next section.

\section{Centers Classification}

This section is devoted to proving the center classification statements of Theorems 1.1 and 1.2. Because the proof of the second result is quite long we have written it separately in Propositions 4.1 and 4.2. The obtained first integrals are globally defined and their level curves provide, among may be others, the three period annuli.

4.1. Proving the center classification in the aligned case. The reversibility property and the canonical form (4) ensures that the origin is a center. Then, by the symmetry, we only need to study the equilibrium point $(0,1)$. First we check that the two families of the statement satisfy the center conditions, and second we compute the explicit expressions of the first integrals, which are of Darboux type, $H_{1}=f_{1}^{\lambda_{1}} f_{2}^{\lambda_{2}}\left(\mathrm{e}^{g}\right)^{\mu}$ and $H_{2}=f_{1}^{\lambda_{1}}\left(\mathrm{e}^{g}\right)^{\mu}$. In both cases $\mu=1$. We will denote by $K_{f_{i}}, i=1,2$, and $K_{g}$ the corresponding cofactors.

The change given by expression (10) allows us to use the algorithm described in Section 2.1 to compute the first center conditions, that is, the first Lyapunov quantities. Although they are polynomials in the parameters, the change (10) introduces some denominators. Straightforward computations show that the denominators of $L_{2}$ and $L_{3}$ before the usual simplifications are $a^{4}\left(a^{2}+4 c^{2}\right)^{2}$ and $a^{8}\left(a^{2}+4 c^{2}\right)^{5}$, respectively, which 
never vanish because $a>0$. Hence, we can consider only the numerators, modulo positive constants,

$$
\begin{aligned}
L_{1}= & c\left(a^{2} b^{2}+2 a^{2} b c+4 b^{2} c^{2}+8 b c^{3}-2 a^{2}+8 c^{2}-4\right), \\
L_{2}= & -c\left(a^{2}+4 c^{2}+2\right)\left(a^{2}+8 b c-4 c^{2}+2\right), \\
L_{3}= & c^{5}\left(a^{2}+4 c^{2}+2\right)\left(a^{2}-4 c^{2}+2\right)\left(6220800 a^{2} c^{14}+24883200 c^{16}+8121600 a^{2} c^{12}\right. \\
& +65664000 c^{14}+3124800 a^{2} c^{10}+51206400 c^{12}+290800 a^{2} c^{8}+13476800 c^{10} \\
& \left.-11200 a^{2} c^{6}+620800 c^{8}+600 a^{2} c^{4}-36000 c^{6}-52 a^{2} c^{2}+1840 c^{4}-a^{2}+60 c^{2}\right) .
\end{aligned}
$$

These values for $L_{i}$ are defined when the previous are zero. Moreover, $L_{4}, L_{5}$ also vanish if $L_{1}=L_{2}=L_{3}=0$. The necessary part of the statement follows easily checking that the real solutions of $\left\{L_{1}=L_{2}=L_{3}=0\right\}$ are the families $\mathcal{A}_{1}$ and $\mathcal{A}_{2}$.

For the sufficient conditions we only list the functions to get the Darboux first integrals for both families.

$\circ$ Family $\mathcal{A}_{1}$ satisfies $c=0$, then

$$
\begin{aligned}
f_{1} & =-2+\left(a^{2}+2\right) y^{2}, \\
f_{2} & =b x+1, \\
g & =-b\left(a^{2}+2\right)\left(\left(a^{2}+2\right) x+y^{2} b\right), \\
K_{f_{1}} & =-\left(a^{2}+2\right) x y, \\
K_{f_{2}} & =b\left(y^{2}-1\right) y, \\
K_{g} & =b\left(a^{2}+2\right)\left(a^{2} b x-\left(a^{2}+2\right) y^{2}+a^{2}+2\right) y,
\end{aligned}
$$

with $\lambda_{1}=a^{2} b^{2}$ and $\lambda_{2}=\left(a^{2}+2\right)^{2}$.

$\circ$ Family $\mathcal{A}_{2}$ satisfies $b=0$ and $c=\sqrt{a^{2}+2}$, then

$$
\begin{aligned}
f_{1} & =-1-2 \sigma x+2\left(a^{2}+2\right) y^{2}+2 \sigma\left(a^{2}+2\right) x y^{2}-\left(a^{2}+2\right) y^{4}, \\
g & =-2 \sigma x, \\
K_{f_{1}} & =-2 \sigma y\left(1+\sigma x-y^{2}\right), \\
K_{g} & =2 \sigma y\left(1+\sigma x-y^{2}\right),
\end{aligned}
$$

with $\sigma=\sqrt{a^{2}+2}$ and $\lambda_{1}=1$.

This, together with the canonical forms derived in Section 3, finishes the proof of Theorem 1.1.

\subsection{Proving the center classification in the triangular case.}

Proposition 4.1. If the equilibrium point $(-1, \pm d)$ of the quartic system (5) is a center then the parameters $(a, b, c, d)$ satisfy one of the conditions given in the statement of Theorem 1.2.

Proof. As the system (5) is reversible with respect to the $x$-axis, the center conditions for the point $(-1,-d)$ are the same as for $(-1, d)$. Thus, we only study this point. From the canonical form computations done in Section $3,(-1, d)$ is a nondegenerate equilibrium point of center-focus type, that is, the trace and the determinant of the Jacobian matrix are zero and positive, respectively.

The proof follows computing a few first Lyapunov quantities and then solving the obtained system to check that all the families are the ones described in the statement.

First, we translate the point to the origin, we do an affine change of coordinates, see (11), and rescaling time. Then, we follow the approach described in Section 2.1 for 
the computation of the center conditions $L_{i}$ in (9). From the affine change, they are rational functions with denominators of the form $c^{k} d^{\ell}$. As usual, the four parameters, $(a, b, c, d)$, appearing in system (5) indicate that only the first four Lyapunov quantities will be necessary to be computed, but as we will see, we use the first five. Instead of the complete expression, for simplicity, we denote by $L_{i}$ only the numerators of the center conditions, that are polynomials with integer coefficients in $(a, b, c, d)$. Because of the size of them, we only show the first one,

$$
\begin{aligned}
L_{1}= & \left(c d^{2}+d^{3}-4 d\right) b^{4}+\left(-8+d^{4}+2 c d^{3}+\left(c^{2}-2\right) d^{2}-4 c d\right) b^{3}+\left(a^{2} c d^{2}+a^{2} d^{3}\right. \\
& \left.-2 a^{2} d-12 c\right) b^{2}+\left(a^{2} d^{4}+2 a^{2} c d^{3}+a^{2}\left(c^{2}-2\right) d^{2}-8 a^{2}-4 c^{2}\right) b+2 a^{4} d-4 a^{2} c .
\end{aligned}
$$

The other four numerators are polynomials of degrees 19,33, 49, and 67 with 317,1524 , 4835, and 12152 monomials, respectively.

For the second step, we need to solve the algebraic system of equations $\mathcal{S}=\left\{L_{1}=L_{2}=\right.$ $\left.L_{3}=L_{4}=L_{5}=0\right\}$. Although this system has only four variables and five equations the usual mechanisms for solving it fails. Instead of finding the solutions directly, we will use the Gianni-Trager-Zacharias algorithm, see [15], to determine the irreducible components of the variety $\mathbf{V}=\mathbf{V}\left(L_{1}, L_{2}, L_{3}, L_{4}, L_{5}\right)$. The main function used is minAssGTZ, it is implemented in the library primdec.lib included on the algebraic computational system SINGULAR, see $[9,10]$. However using the function directly we were not able to compute the decomposition neither over the field of rational numbers nor in the finite field $\mathbb{Z}_{p}$, with $p=32003$. Hence, the irreducible components will be computed considering some auxiliary polynomials obtained from some crossed resultants with respect to one of the parameters, because the new polynomials have less variables (but higher degree) and contain the solutions that we are interested.

Thus, we compute the four resultants with respect to the parameter $c, \operatorname{Res}\left(L_{1}, L_{2}, c\right)$, $\operatorname{Res}\left(L_{1}, L_{3}, c\right), \operatorname{Res}\left(L_{1}, L_{4}, c\right)$, and $\operatorname{Res}\left(L_{1}, L_{5}, c\right)$. We denote by $\operatorname{Res}_{12}, \operatorname{Res}_{13}, \operatorname{Res}_{14}$, and Res $_{15}$, the corresponding factorized expressions, leaving only one term, when they have multiplicity bigger than one, and removing the nonvanishing terms, that is the powers of $a, d$, and $a^{2}+b^{2}$. Then, we get

$$
\operatorname{Res}_{12}=\mathcal{R} \cdot \mathcal{R}_{12}, \quad \operatorname{Res}_{13}=\mathcal{R} \cdot \mathcal{R}_{13}, \quad \operatorname{Res}_{14}=\mathcal{R} \cdot \mathcal{R}_{14}, \quad \operatorname{Res}_{15}=\mathcal{R} \cdot \mathcal{R}_{15},
$$

with the common factor $\mathcal{R}=\mathcal{R}_{1} \mathcal{R}_{2} \cdots \mathcal{R}_{8}$, where

$$
\begin{aligned}
& \mathcal{R}_{1}=b, \\
& \mathcal{R}_{2}=b d+2, \\
& \mathcal{R}_{3}=a^{2} d^{2}+b^{2} d^{2}-2 b d+2 d^{2}-8, \\
& \mathcal{R}_{4}=a^{2} d+b^{2} d-b d^{2}+2 b, \\
& \mathcal{R}_{5}=a^{2} d-b^{2} d-2 b+2 d, \\
& \mathcal{R}_{6}=a^{2} d^{2}+b^{2} d^{2}-4, \\
& \mathcal{R}_{7}=a^{2}-b d, \\
& \mathcal{R}_{8}=a^{2} d^{2}+(b d+2)^{2}+2 d^{2} .
\end{aligned}
$$

We have not written, because of their size, the polynomials $\mathcal{R}_{12}, \mathcal{R}_{13}, \mathcal{R}_{14}, \mathcal{R}_{15}$. They have degrees $6,32,62,92$ in $(a, b, d)$ and 9, 293, 1642, and 4803 monomials, respectively.

With the above discussion, and taking into account that the surface $\mathcal{R}_{8}=0$ has no real points because $a, d>0$, the solution of $\mathcal{S}$ can be studied solving the eight algebraic systems $\mathcal{S}_{0}=\left\{L_{1}=L_{2}=L_{3}=L_{4}=L_{5}=\mathcal{R}_{12}=\mathcal{R}_{13}=\mathcal{R}_{14}=\mathcal{R}_{15}=0\right\}$ and $\mathcal{S}_{i}=\left\{L_{1}=L_{2}=L_{3}=L_{4}=\mathcal{R}_{i}=0\right\}$ for $i=1, \ldots, 7$. 
System $\mathcal{S}_{0}$ is studied computing the two by two crossed resultants, first with respect to $d$, then with respect to $b$. In each step we remove, as previously, all the multiple factors and also the powers of the nonvanishing variables or factors $\left(d a, a^{2}+b^{2}, a^{2}+2\right)$. Finally, the last two polynomials have only one common factor $a^{2}-1$. Next, as $a>0$, we consider the system $\left\{\mathcal{R}_{12}=\mathcal{R}_{13}=\mathcal{R}_{14}=\mathcal{R}_{15}=a-1=0\right\}$, that has, since $d>0$, only one solution $\{a=1, b=1 / \sqrt{3}, d=\sqrt{3}\}$. Finally, $\mathcal{S}_{0}$ has only two real solutions $\{a=1, b=1 / \sqrt{3}, c=2 / \sqrt{3}, d=\sqrt{3}\}$ and $\{a=1, b=1 / \sqrt{3}, c=-4 / \sqrt{3}, d=\sqrt{3}\}$. They, satisfy the conditions of cases $\mathcal{T}_{17}$ and $\mathcal{T}_{5}$ of the statement, respectively.

Although some systems can be easily solved, as $\mathcal{S}_{1}$ that writes equivalently as the first family, $\left\{b=a^{2} d-2 c=0\right\}$, most of them need an accurate procedure. We will follow step by step the algorithm described in [26].

The 18 families in the statement are obtained solving systems $\mathcal{S}_{i}$, for $i=1, \ldots, 7$.

For each $i$, except for $i=4$, we use the mentioned Gianni-Trager-Zacharias algorithm and we apply the routine minAssGTZ to the ideal $\mathcal{B}_{i}=\left\langle\mathcal{R}_{i}, L_{1}, L_{2}, L_{3}, L_{4}\right\rangle$, for simplicity we have not used $L_{5}$. That is, we obtain the necessary conditions to have a center finding the irreducible decomposition of the variety of each ideal $\mathcal{B}_{i}$. We illustrate the procedure only for one family, $\mathcal{S}_{7}$, the other follow similarly.

Working in $\mathbb{Z}_{p}[a, b, c, d]$, with $p=32003$, instead of $\mathbb{Q}[a, b, c, d]$, the minimal associate prime ideals of $\mathcal{B}_{7}$, provided by SINGULAR, are

$$
\begin{gathered}
\langle a, b\rangle,\langle a, d, b-16001 c\rangle, \\
\left\langle c d+2 d^{2}-8, b+16001 c, a^{2}+d^{2}-4\right\rangle,\left\langle c d-d^{2}-2, b+c, a^{2}+d^{2}+2\right\rangle, \\
\left\langle d^{3}+c-2 d, b+d, a^{2}+d^{2}\right\rangle,\left\langle a^{2}+c d+d^{2}, b+c+d\right\rangle .
\end{gathered}
$$

Next, we use the rational reconstruction algorithm provided by [34] to get a candidate to be the minimal associate prime ideal but with rational coefficients. In fact, we should apply the next function to each coefficient of the above polynomials.

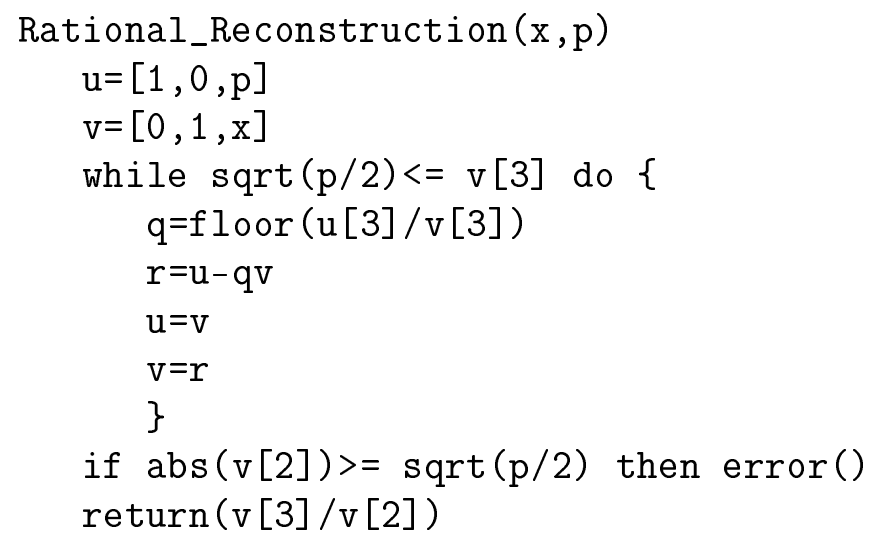

We recall that the floor function, $\lfloor x\rfloor$, gives the greatest integer less than or equal to $x$. Given an integer $x$ and a prime $p$, the rational reconstruction function defined above, provides a rational number $y$ such that $y=x(\bmod p)$ and, in absolute value, the numerator and denominator of $y$ are less than $\sqrt{p / 2}$.

In the primary decomposition ideals of $\mathcal{B}_{7}$ given in (12) all the rational reconstructed values are the same integers except 16001 , which is $-1 / 2$. Consequently, the candidate 
to be the minimal associate prime ideals of $\mathcal{B}_{7}$ are

$$
\begin{aligned}
\widetilde{P}_{1} & =\langle a, b\rangle, \\
\widetilde{P}_{2} & =\langle a, d, b+(1 / 2) c\rangle, \\
\widetilde{P}_{3} & =\left\langle c d+2 d^{2}-8, b-(1 / 2) c, a^{2}+d^{2}-4\right\rangle, \\
\widetilde{P}_{4} & =\left\langle c d-d^{2}-2, b+c, a^{2}+d^{2}+2\right\rangle, \\
\widetilde{P}_{5} & =\left\langle d^{3}+c-2 d, b+d, a^{2}+d^{2}\right\rangle, \\
\widetilde{P}_{6} & =\left\langle a^{2}+c d+d^{2}, b+c+d\right\rangle .
\end{aligned}
$$

The next step is to show that $\sqrt{P_{7}}=\sqrt{\mathcal{B}_{7}}$, where $P_{7}=\bigcap_{k=1}^{6} \widetilde{P}_{k}$ in $\mathbb{Q}[a, b, c, d]$. We have denoted by $\sqrt{P}$ the radical of the ideal $P$. In general, it is simpler to verify the double inclusion instead of computing the radicals. Adding a new artificial parameter $w$, this property can be seen checking that $\{1\}$ is the Gröbner basis of the next list of ideals, $\left\langle 1-w L_{k}, P_{7}\right\rangle$, for $k=1, \ldots, 4$ and $\left\langle 1-w p, \mathcal{B}_{7}\right\rangle$, for every $p \in P_{7}$.

In the last step we check which $\widetilde{P}_{k}$, for $k=1, \ldots, 6$, will appear in the statement. As $a, d, a^{2}+d^{2}, a^{2}+d^{2}+2$ are nonvanishing, only $\widetilde{P}_{3}$ and $\widetilde{P}_{6}$ are necessary conditions for system (5) be a center. In fact, they are cases $\mathcal{T}_{17}$ and $\mathcal{T}_{18}$, respectively.

Finally, we study the family $\mathcal{S}_{4}=\left\{\mathcal{R}_{4}, L_{1}, L_{2}, L_{3}, L_{4}\right\}$ with another procedure. Because, for the rational reconstructed candidate to be the primary decomposition ideals we have $\sqrt{P_{4}} \neq \sqrt{\mathcal{B}_{4}}$. As the condition $b=0$ has been studied before, here we will assume that $b \neq 0$.

We start again computing the four resultants but with respect to the parameter $a$,

$$
\operatorname{Res}\left(\mathcal{R}_{4}, L_{1}, a\right), \quad \operatorname{Res}\left(\mathcal{R}_{4}, L_{2}, a\right), \quad \operatorname{Res}\left(\mathcal{R}_{4}, L_{3}, a\right), \quad \operatorname{Res}\left(\mathcal{R}_{4}, L_{4}, a\right) .
$$

We denote by $\widehat{\operatorname{Res}}_{1}, \widehat{\operatorname{Res}}_{2}, \widehat{\operatorname{Res}}_{3}$, and $\widehat{\operatorname{Res}}_{4}$, the corresponding factorized expressions. But leaving only one term, when they have multiplicity bigger than one, and removing the nonvanishing terms, that are powers of $b, d$. Then, we get

$$
\widehat{\operatorname{Res}}_{1}=\widehat{\mathcal{R}} \cdot \widehat{\mathcal{R}}_{1}, \quad \widehat{\operatorname{Res}}_{2}=\widehat{\mathcal{R}} \cdot \widehat{\mathcal{R}}_{2}, \quad \widehat{\operatorname{Res}}_{3}=\widehat{\mathcal{R}} \cdot \widehat{\mathcal{R}}_{3}, \quad \widehat{\operatorname{Res}}_{4}=\widehat{\mathcal{R}} \cdot \widehat{\mathcal{R}}_{4},
$$

with the common factor $\widehat{\mathcal{R}}=c d+d^{2}-2$. Hence, the solution $\left\{L_{1}=L_{2}=L_{3}=L_{4}=\right.$ $\left.\widehat{\mathcal{R}}_{4}=\widehat{\mathcal{R}}=0\right\}$ is case $(7)$.

As we have reduced the set of variables to $(b, c, d)$, we can follow as before solving the system $\left\{\widehat{\mathcal{R}}_{1}=\widehat{\mathcal{R}}_{2}=\widehat{\mathcal{R}}_{3}=\widehat{\mathcal{R}}_{4}=0\right\}$ by computing the crossed resultants $\operatorname{Res}\left(\widehat{\mathcal{R}}_{1}, \widehat{\mathcal{R}}_{2}, b\right)$, $\operatorname{Res}\left(\widehat{\mathcal{R}}_{1}, \widehat{\mathcal{R}}_{3}, b\right)$, and $\operatorname{Res}\left(\widehat{\mathcal{R}}_{1}, \widehat{\mathcal{R}}_{4}, b\right)$. Now, leaving also only one term, when they have multiplicity bigger than one, and removing the nonvanishing terms, that are powers of $c, d$, $d^{2}+2, d+2$, and $c d+d^{2}-2$, we get

$$
\widehat{\mathcal{R}}_{12}=\widetilde{\mathcal{R}} \cdot \widetilde{\mathcal{R}}_{12}, \quad \widehat{\mathcal{R}}_{13}=\widetilde{\mathcal{R}} \cdot \widetilde{\mathcal{R}}_{13}, \quad \widehat{\mathcal{R}}_{14}=\widetilde{\mathcal{R}} \cdot \widetilde{\mathcal{R}}_{14},
$$

where $\widetilde{\mathcal{R}}=(d-2)\left(d^{2}-2\right)(2 c+3 d)\left(c d+2 d^{2}-4\right)\left(d^{2}-6\right)$. As $\widetilde{\mathcal{R}}_{12}=d^{2}+6 \neq 0$, we have to check that, for each factor $D$ in $\widetilde{\mathcal{R}}$, the solution of $\mathcal{S}_{D}=\left\{L_{1}=L_{2}=L_{3}=L_{4}=\mathcal{R}_{4}=D=0\right\}$ is in one of the cases listed in the statement. For example, for $d=2$, we obtain the two solutions $\left\{a^{2}+b^{2}-b=c+1=d-2=0\right\}$ and $\left\{a^{2}+b^{2}-b=c-b=d-2=0\right\}$. The first is (4) and the second is (8). The other factors are found similarly.

Proposition 4.2. For each family in the statement of Theorem 1.2, the quartic system (5), which is reversible with respect to the $x$-axis, has a center at the origin and two centers at the points $(-1, \pm d)$. 
Proof. The origin of (5) is a nondegenerate center-focus point then, by the symmetry property, it is a center. The points $(-1, \pm d)$ are also nondegenerate equilibria of centerfocus type. The proof follows straightforward doing a case by case study. We compute the first integrals, which are of Darboux type, and check that all of them are well defined in a neighborhood of such equilibrium points. In particular, there are only four types of first integrals:

$$
H_{1}=f_{1}, \quad H_{2}=f_{1}^{\lambda_{1}} f_{2}^{\lambda_{2}}, \quad H_{3}=f_{1}^{\lambda_{1}}\left(\mathrm{e}^{g}\right)^{\mu}, \quad \text { and } \quad H_{4}=f_{1}^{\lambda_{1}} f_{2}^{\lambda_{2}}\left(\mathrm{e}^{g}\right)^{\mu} .
$$

In the rest of the proof we only show the explicit expressions of the polynomials $f_{1}, f_{2}, g$ and the real numbers $\lambda_{1}, \lambda_{2}$, because $\mu=1$. We also list the associated cofactors $K_{f_{1}}, K_{f_{2}}$, and $K_{g}$, when their expressions are not so big. We recall that $f_{1}=0$ and $f_{2}=0$ are invariant algebraic curves and $\mathrm{e}^{g}$ is an exponential factor.

The cases are listed grouped by the expression of the corresponding first integral.

- Cases corresponding to the Hamiltonian systems with the first integral $H_{1}=f_{1}$ :

$\left(\mathcal{T}_{3}\right)$ In this case, we have $a^{2}=-\left(d^{2} c^{2}+2 d\left(d^{2}-5\right) c+\left(d^{2}-4\right)^{2}\right)$ and $b=\left(c d+d^{2}-4\right) / d$. Then, as $a^{2}$ should be nonnegative, we have that $\left(-d^{2}+5-\sqrt{-2 d^{2}+9}\right) / d<c<\left(-d^{2}+\right.$ $\left.5+\sqrt{-2 d^{2}+9}\right) / d$, and $0<d<3 \sqrt{2} / 2$. We remark that system (5) depends quadratically in $a$. With these conditions, we get

$$
f_{1}=2 d^{4} x^{2}+2 d^{4} y^{2}+2 d^{3}(c+d) x y^{2}-\left(c d+2 d^{2}-4\right) y^{4}-2\left(c d+d^{2}-2\right) x y^{4} .
$$

$\left(\mathcal{T}_{8}\right)$ Here $a^{2}=c-c^{2}, b=c$, and $d=2$. Hence, when $0<c<1$, system (5) is well defined. Then,

$$
f_{1}=16 x^{2}+16 y^{2}+8(c+2) x y^{2}-(c+2) y^{4}-2(c+1) x y^{4} .
$$

$\left(\mathcal{T}_{12}\right)$ In this case, we have $a^{2}=2\left(4-d^{2}\right) / d^{2}, b=2 / d$ and $c=\left(6-d^{2}\right) / d$. Thus, when $0<d<2$, system (5) is well defined and we get

$$
f_{1}=2 d^{4} x^{2}+2 d^{4} y^{2}+12 d^{2} x y^{2}-\left(d^{2}+2\right) y^{4}-8 x y^{4} .
$$

$\left(\mathcal{T}_{14}\right)$ Here, we have $a^{2}=4-d^{2}, b=\left(d^{2}-2\right) / d$ and $c=2 / d$. So, the condition $0<d<2$ is necessary and we have

$$
f_{1}=d^{4} x^{2}+d^{4} y^{2}+\left(d^{2}+2\right) d^{2} x y^{2}-\left(d^{2}-1\right) y^{4}-d^{2} x y^{4} .
$$

$\left(\mathcal{T}_{17}\right)$ In this case, we have $a^{2}=4-d^{2}, b=\left(4-d^{2}\right) / d$, and $c=2\left(4-d^{2}\right) / d$. Here, $0<d<2$ and

$$
f_{1}=d^{4} x^{2}+d^{4} y^{2}-\left(d^{2}-8\right) d^{2} x y^{2}-2 y^{4}+\left(d^{2}-6\right) x y^{4} .
$$

○ Cases corresponding to $H_{2}=f_{1}^{\lambda_{1}} f_{2}^{\lambda_{2}}$ :

$\left(\mathcal{T}_{1}\right)$ We can write $b=0$ and $c=a^{2} d / 2$, for obtaining

$$
\begin{aligned}
f_{1} & =2 d^{6}-8 d^{4} x-\left(2 a^{2} d^{2}+4 d^{2}+16\right) d^{2} y^{2}+\left(a^{2} d^{2}+2 d^{2}+8\right) y^{4}, \\
f_{2} & =2+\left(a^{2}+2\right) x, \\
K_{f_{1}} & =\frac{4}{d^{4}}\left(d^{2}-y^{2}\right) y, \\
K_{f_{2}} & =-\frac{\left(a^{2}+2\right)}{2 d^{2}}\left(d^{2}-y^{2}\right) y,
\end{aligned}
$$

with $\lambda_{1}=d^{2}\left(a^{2}+2\right), \lambda_{2}=8$. 
$\left(\mathcal{T}_{16}\right)$ Here we have $a=2 / d, b=0$, and $c=2 / d$. Then, we get $\lambda_{1}=1, \lambda_{2}=4 /\left(d^{2}+2\right)$, and

$$
\begin{aligned}
f_{1} & =d^{6}-4 d^{4} x-2\left(d^{2}+6\right) d^{2} y^{2}+\left(d^{2}+6\right) y^{4}, \\
f_{2} & =d^{2}+\left(d^{2}+2\right) x, \\
K_{f_{1}} & =\frac{4\left(d^{2}-y^{2}\right) y}{d^{4}}, \\
K_{f_{2}} & =-\frac{\left(d^{2}+2\right)\left(d^{2}-y^{2}\right) y}{d^{4}} .
\end{aligned}
$$

○ Cases corresponding to $H_{3}=f_{1}^{\lambda_{1}}\left(\mathrm{e}^{g}\right)^{\mu}$, with $\mu=1$ :

$\left(\mathcal{T}_{2}\right)$ In this case we have $b=-2 / d$ and

$$
-a^{2} d^{2} c^{2}-2 a^{2} c d^{4}-8 c d^{2}+a^{4} d^{2}-a^{2} d^{4}+4 a^{2} d^{2}+4 a^{2}-4 d^{2}+16=0 .
$$

As $a>0$, the discriminant $\sigma=\left(a^{2}+2\right)^{2}\left(a^{2} d^{2}+4\right)$ is positive for all $a, d$. Adding an artificial variable, $e$, the curve $\sigma-e^{2}=\left(a^{2}+2\right)^{2} a^{2} d^{2}-e^{2}+\left(2 a^{2}+4\right)^{2}=0$ is a hyperbola with respect to the variables $(d, e)$. Then, we can find a rational parameterization

$$
d=\frac{4\left(a^{2}+2\right)^{2}-t^{2}}{2 a t\left(a^{2}+2\right)}
$$

such that $\sigma$ is a perfect square. Hence, we can isolate $b, c$ in terms of $a, t$ :

$$
\begin{aligned}
& c(a, t)=-\frac{\left(t^{2}+2\left(a^{2}+2\right)^{2} t+4\left(a^{2}+2\right)^{2}\right)\left(t^{2}+4\left(a^{2}+2\right)^{2}\right)}{2 a t\left(4\left(a^{2}+2\right)^{2}-t^{2}\right)\left(a^{2}+2\right)}, \\
& b(a, t)=\frac{4 a t\left(a^{2}+2\right)}{t^{2}-4\left(a^{2}+2\right)^{2}} .
\end{aligned}
$$

Then, we get $\lambda_{1}=-1$ and

$$
\begin{aligned}
f_{1}= & 4\left(a^{2}+2\right)^{5} t^{3}\left(2 a^{2}+t+4\right)^{2} a^{2} x y^{2} \\
& -2\left(a^{2}+2\right)^{3} a^{2} t^{2}\left(t^{4}-8\left(a^{2}+2\right)^{2}\left(a^{2}+1\right) t^{2}-16\left(a^{2}+2\right)^{4}\right) y^{2} \\
& -2\left(a^{2}+2\right)^{2} t\left(4\left(a^{2}+2\right)^{2}-t^{2}\right)^{2}\left(t^{2}+2\left(a^{2}+2\right)^{2} t+4\left(a^{2}+2\right)^{2}\right) x \\
& +\left(2 a^{2}-t+4\right)^{4}\left(t^{2}+2\left(a^{2}+2\right)^{2} t+4\left(a^{2}+2\right)^{2}\right)^{2}, \\
g= & \frac{4\left(a^{2}+2\right)^{3} t^{2} y^{2}}{\left(2 a^{2}-t+4\right)^{4}}-\frac{2\left(a^{2}+2\right)^{2}\left(2 a^{2}+t+4\right)^{2} t x}{\left(2 a^{2}-t+4\right)^{2}\left(t^{2}+2\left(a^{2}+2\right)^{2} t+4\left(a^{2}+2\right)^{2}\right)} .
\end{aligned}
$$

We remark that without the parameterization the expressions for $f_{1}$ and $g$ are more difficult to be found.

$\left(\mathcal{T}_{4}\right)$ Here $a^{2}=b(1-b), c=-1$ and $d=2$. So, system (5) is well defined only when $0<b<1$. Moreover, $\lambda_{1}=4 b$ and

$$
\begin{aligned}
f_{1} & =-4+(b+1) y^{2}, \\
g & =-(b+1)\left((2 b+2) x^{2}+(b+2) y^{2}+(b+1) x y^{2}\right), \\
K_{f_{1}} & =-\frac{(b+1)\left(y^{2}+4 x\right) y}{8} \\
K_{g} & =\frac{b(b+1)\left(y^{2}+4 x\right) y}{2} .
\end{aligned}
$$


$\left(\mathcal{T}_{6}\right)$ For this case we have $a^{2}=2\left(4-d^{2}\right) / d^{2}, b=2 / d$, and $c=-\left(d^{2}+2\right) / d$. So, when $0<d<2$ system (5) is well defined. Additionally,

$$
\begin{aligned}
f_{1} & =d^{4}\left(2+d^{2}\right)^{2}-8 d^{4}\left(d^{2}+2\right) x-32 d^{4} y^{2}+64 d^{2} x y^{2}+16\left(d^{2}+2\right) y^{4}, \\
g & =\frac{8 x}{d^{2}+2}, \\
K_{f_{1}} & =\frac{8\left(d^{4}-2 d^{2} x-\left(d^{2}+2\right) y^{2}\right) y}{\left(d^{2}+2\right) d^{4}} \\
K_{g} & =-\frac{8\left(d^{4}-2 d^{2} x-\left(d^{2}+2\right) y^{2}\right) y}{\left(d^{2}+2\right) d^{4}} .
\end{aligned}
$$

and $\lambda_{1}=1$.

$\left(\mathcal{T}_{7}\right)$ Here, $a^{2}=b\left(d^{2}-b d-2\right) / d$ and $c=-\left(d^{2}-2\right) / d$. There are two cases such that system (5) is well defined: (i) $b>0, d<\left(b-\sqrt{b^{2}+8}\right) / 2$ or $\left(b+\sqrt{b^{2}+8}\right) / 2<d$, and (ii) $b<0,\left(b-\sqrt{b^{2}+8}\right) / 2<d<\left(b+\sqrt{b^{2}+8}\right) / 2$. Independently, we have

$$
\begin{aligned}
f_{1} & =-2 d^{2}+(b d+2) y^{2}, \\
g & =-(b d+2)\left(\left(b d^{3}+2 d^{2}\right) x^{2}+\left(2 b d+2 d^{2}\right) y^{2}+(2 b d+4) x y^{2}\right), \\
K_{f_{1}} & =-\frac{(b d+2)\left(y^{2}+d^{2} x\right) y}{d^{4}}, \\
K_{g} & =\frac{2 b\left(d^{2}-2\right)(b d+2)\left(y^{2}+d^{2} x\right) y}{d},
\end{aligned}
$$

with $\lambda_{1}=2 b d^{3}\left(d^{2}-2\right)$.

$\left(\mathcal{T}_{9}\right)$ In this case, $a^{2}=\left(d^{2}-4\right) / 4, b=d / 2$, and $c=-3 d / 2$. Hence, when $d>2$ system $(5)$ is well defined. Here we get

$$
\begin{aligned}
f_{1}= & 18 d^{6}-\left(12 d^{6}+48 d^{4}\right) x-\left(4 d^{6}+32 d^{4}+64 d^{2}\right) y^{2}+\left(2 d^{6}+16 d^{4}+32 d^{2}\right) x y^{2} \\
& +\left(3 d^{4}+24 d^{2}+48\right) y^{4}, \\
g_{1}= & 2 x\left(d^{2}+4\right) / 3 d^{2}, \\
K_{f_{1}}= & \frac{\left(d^{2}+4\right)\left(3 y^{3}+d^{2} x-2 d^{2}\right) y}{d^{4}}, \\
K_{g}= & -\frac{\left(d^{2}+4\right)\left(3 y^{3}+d^{2} x-2 d^{2}\right) y}{d^{4}},
\end{aligned}
$$

with $\lambda_{1}=1$.

$\left(\mathcal{T}_{10}\right)$ The discriminant with respect to $c$ of the second condition writes as $\sigma=4\left(a^{2} d^{2}+\right.$ $\left.2 d^{2}+1\right)$. Similarly, as for family $\mathcal{T}_{2}$, adding an artificial variable, $e$, from the rational parameterization of the hyperbola $\sigma-e^{2}=0$, we know that

$$
a=\frac{-2 d^{2}+t^{2}-1}{2 d t}
$$

Then, the second condition allow us to write

$$
\begin{aligned}
& b=\frac{2 d^{2}+t^{2}-2 t+1}{2 d t}, \\
& c=-\frac{2 d^{2} t+2 d^{2}+t^{2}-2 t+1}{2 d t} .
\end{aligned}
$$


In fact, the other solution is equivalent to changing $t$ by $-t$, moreover system (5) depends on $a^{2}$. Hence,

$$
\begin{aligned}
f_{1}= & 2 d^{4} t\left(2 d^{2}(t+1)+(t-1)^{2}\right)^{2}-4 d^{4} t\left(2 d^{2}+(t+1)^{2}\right)\left(2 d^{2}(t+1)+(t-1)^{2}\right) x \\
& -4 d^{4} t\left(2 d^{2}+(t+1)^{2}\right)^{2} y^{2}+2 d^{2}\left(2 d^{2}+(t-1)^{2}\right)\left(2 d^{2}+(t+1)^{2}\right)^{2} x y^{2} \\
& +\left(2 d^{2}(t+1)+(t-1)^{2}\right)\left(2 d^{2}+(t+1)^{2}\right)^{2} y^{4} \\
g= & \frac{2\left(2 d^{2}+(t+1)^{2}\right) x}{2 d^{2}(t+1)+(t-1)^{2}}, \\
K_{f_{1}}= & \frac{\left(2 d^{2}+(t+1)^{2}\right)\left(2 d^{4} t-d^{2}\left(2 d^{2}+(t-1)^{2}\right) x-\left(2 d^{2}\left(t y^{2}+1\right)+(t-1)^{2}\right) y^{2}\right) y}{t d^{4}\left(2 d^{2}(t+1)+(t-1)^{2}\right)}, \\
K_{g}= & -\frac{\left(2 d^{2}+(t+1)^{2}\right)\left(2 d^{4} t-d^{2}\left(2 d^{2}+(t-1)^{2}\right) x-\left(2 d^{2}\left(t y^{2}+1\right)+(t-1)^{2}\right) y^{2}\right) y}{t d^{4}\left(2 d^{2}(t+1)+(t-1)^{2}\right)} .
\end{aligned}
$$

with $\lambda_{1}=1$.

$\left(\mathcal{T}_{11}\right)$ In this case, as $d>0$, we have $b=\sqrt{a^{2}+1}, c=-\left(2 a^{2}+1\right) / \sqrt{a^{2}+1}$, and $d=$ $2 \sqrt{a^{2}+1}$. Then,

$$
\begin{aligned}
f_{1} & =-4\left(1+a^{2}\right)+\left(a^{2}+2\right) y^{2}, \\
g & =\left(a^{2}+2\right)\left(\left(2 a^{4}+6 a^{2}+4\right) x^{2}+\left(a^{2}+2\right) x y^{2}+\left(3 a^{2}+3\right) y^{2}\right), \\
K_{f_{1}} & =-\frac{\left(a^{2}+2\right)\left(\left(4 a^{2}+4\right) x+y^{2}\right) y}{8\left(a^{2}+1\right)^{2}} \\
K_{g} & =-\frac{\left(2 a^{2}+1\right)\left(a^{2}+2\right)\left(\left(4 a^{2}+4\right) x+y^{2}\right) y}{2},
\end{aligned}
$$

with $\lambda_{1}=-4\left(2 a^{2}+1\right)\left(a^{2}+1\right)^{2}$.

$\left(\mathcal{T}_{13}\right)$ Here we have $b=4 / d\left(d^{2}-2\right), c=-\left(d^{2}-2\right) / d$, and $\left(d^{2}-2\right)^{2} a^{2}-4\left(d^{2}-4\right)=0$.

Last condition can be written equivalently as the rational parameterization

$$
(a, d)=\left(\frac{8(2 t-1)(2 t+1) t}{16 t^{4}+24 t^{2}+1},-\frac{2\left(4 t^{2}+1\right)}{(2 t-1)(2 t+1)}\right) .
$$

This expression simplifies the writing of

$$
\begin{aligned}
f_{1}= & -32 t^{4}-48 t^{2}-2+\left(16 t^{4}-8 t^{2}+1\right) y^{2}, \\
g= & -\left(512 t^{8}+512 t^{6}+192 t^{4}+32 t^{2}+2\right) x^{2}-\left(384 t^{8}+384 t^{6}+272 t^{4}+24 t^{2}+3 / 2\right) y^{2} \\
& -\left(256 t^{8}-32 t^{4}+1\right) x y^{2} \\
K_{f_{1}}= & -\frac{\left(4 t^{2}-1\right)^{2}\left(\left(64 t^{4}+32 t^{2}+4\right) x y+\left(16 t^{4}-8 t^{2}+1\right) y^{3}\right)}{4\left(16 t^{4}+24 t^{2}+1\right)\left(4 t^{2}+1\right)^{2}}, \\
K_{g}= & \frac{\left(4 t^{2}-1\right)^{2}\left(16 t^{4}+24 t^{2}+1\right)\left(\left(64 t^{4}+32 t^{2}+4\right) x y+\left(16 t^{4}-8 t^{2}+1\right) y^{3}\right)}{4\left(4 t^{2}+1\right)^{2}},
\end{aligned}
$$

with $\lambda_{1}=\left(16 t^{4}+24 t^{2}+1\right)^{2}$.

$\left(\mathcal{T}_{15}\right)$ In this case, we have two solutions but, as $a^{2}>0$, only one corresponds to real values:

$$
a^{2}=\frac{-2 d^{2}+\sqrt{4 d^{2}+9}+3}{2 d^{2}}, \quad b=-\frac{1-\sqrt{4 d^{2}+9}}{2 d}, \quad c=\frac{-2 d^{2}+1-\sqrt{4 d^{2}+9}}{2 d} .
$$


Then,

$$
\begin{aligned}
f_{1}= & 2 d^{6}-(\sigma+3) d^{4}+8(\sigma-2) d^{2}-12 \sigma+36-2 d^{2}\left(\sigma d^{2}-d^{2}-2 \sigma+6\right) x \\
& -4 d^{4} y^{2}+2 d^{2}(\sigma-1) x y^{2}+\left(2 d^{2}+\sigma-1\right) y^{4}, \\
g= & \frac{\left((\sigma+1) d^{2}-2 \sigma-6\right) x}{d^{4}-2 d^{2}-2}, \\
K_{f_{1}}= & \frac{\left(d^{4}\left(\sigma d^{2}+d^{2}-2 \sigma-6\right)+2 d^{2}\left(-d^{4}+\sigma+3\right) x-\left(d^{4}-2 d^{2}-2\right)(\sigma+3) y^{2}\right) y}{\left(d^{4}-2 d^{2}-2\right) d^{4}}, \\
K_{g}= & -\frac{\left(d^{4}\left(\sigma d^{2}+d^{2}-2 \sigma-6\right)+2 d^{2}\left(-d^{4}+\sigma+3\right) x-\left(d^{4}-2 d^{2}-2\right)(\sigma+3) y^{2}\right) y}{\left(d^{4}-2 d^{2}-2\right) d^{4}},
\end{aligned}
$$

with $\sigma=\sqrt{4 d^{2}+9}$ and $\lambda_{1}=1$.

○ Cases corresponding to $H_{4}=f_{1}^{\lambda_{1}} f_{2}^{\lambda_{2}}\left(\mathrm{e}^{g}\right)^{\mu}$, with $\mu=1$ :

$\left(\mathcal{T}_{5}\right)$ Here, the parameters satisfy $a^{2}=4-d^{2}, b=\left(4-d^{2}\right) / d$, and $c=-4 / d$. Thus, the condition $0<d<2$ is necessary in order that system $(5)$ is well defined. Then,

$$
\begin{aligned}
f_{1} & =d^{4}+d^{2}\left(d^{2}-6\right) x+\left(d^{2}-6\right) y^{2}, \\
f_{2} & =2 d^{2}+\left(d^{2}-6\right) y^{2} \\
g & =\left(d^{2}-6\right) x \\
K_{f_{1}} & =\frac{\left(d^{2}-6\right)\left(d^{2} x+y^{2}\right) y}{d^{4}} \\
K_{f_{2}} & =-\frac{\left(d^{2}-6\right)\left(d^{2}-y^{2}\right) y}{d^{4}} \\
K_{g} & =-\frac{\left(d^{2}-6\right)\left(d^{4}+\left(d^{2}-4\right) d^{2} x-4 y^{2}\right)}{d^{4}},
\end{aligned}
$$

with $\lambda_{1}=-d^{2}, \lambda_{2}=d^{2}-4$.

$\left(\mathcal{T}_{18}\right)$ In this case, we have $b=a^{2} / d$ and $c=-\left(a^{2}+d^{2}\right) / d$. Then,

$$
\begin{aligned}
f_{1} & =-d^{4}+d^{2}\left(a^{2}+2\right) x+\left(a^{2}+2\right) y^{2}, \\
f_{2} & =-2 d^{2}+\left(a^{2}+2\right) y^{2}, \\
g & =\left(a^{2}+2\right) x \\
K_{f_{1}} & =\frac{\left(a^{2}+2\right)\left(d^{2}-y^{2}\right) y}{d^{4}} \\
K_{f_{2}} & =-\frac{\left(a^{2}+2\right)\left(d^{2} x+y^{2}\right) y}{d^{4}}, \\
K_{g} & =\frac{\left(a^{2}+2\right)\left(-d^{4}+a^{2} d^{2} x+\left(a^{2}+d^{2}\right) y^{2}\right) y}{d^{4}},
\end{aligned}
$$

with $\lambda_{1}=d^{2}, \lambda_{2}=a^{2}$.

\section{Simultaneous Cyclicity}

This section is devoted to studying the simultaneous degenerate Hopf bifurcation of system (5) for different values of the parameters $(a, b, c, d)$. Before stating and proving the main result of this section, we recall in few words the bifurcation scheme presented in [6] for first order perturbations and degree four systems. The unperturbed system (5) has three centers located in the vertex of a triangle. As two of them are symmetric with respect to the $x$-axis, we study only two simultaneous degenerated Hopf bifurcations. The bifurcation will be done in two steps. First, we consider a perturbation which is symmetric 
with respect to the $x$-axis to get $n$ symmetric hyperbolic small limit cycles surrounding the symmetric centers using Theorem 2.3. With this first perturbation, the origin remains a center. Second, we consider a general perturbation to get $m$ small hyperbolic limit cycles surrounding the origin, also by Theorem 2.3. We obtain in total $2 n+m$ small limit cycles in configuration $\langle n, m, n\rangle$.

Recently, the same procedure has been used in [24], but with a higher order development, to some systems with degrees up to ten, improving the best global Hilbert numbers known up to now for polynomial systems from degree four to ten. Here, as in [6], we only study first order developments of the Lyapunov quantities introduced in Section 2.2. The study of higher order perturbations will be done in a near future.

Proposition 5.1. There exist polynomial perturbations of degree four such that at least 13 small limit cycles bifurcate from the centers of system (5). They present at least two different configurations varying the parameters $(a, b, c, d):\langle 4,5,4\rangle$ and $\langle 3,7,3\rangle$. Moreover the phase portraits in the Poincaré disk for the unperturbed systems are also different. See Figure 2.

Proof. The proof follows the bifurcation procedure described in the beginning of this section. We will describe only the statement to get the 13 small limit cycles in configuration $\langle 4,5,4\rangle$, fixing the values of the parameters of the unperturbed center as $(a, b, c, d)=(1 / 2,1 / 2,-1,2)$. It corresponds to family $\mathcal{T}_{4}$. The same result and configuration can be found in family $\mathcal{T}_{7}$, fixing the parameters to $(1 / 2,-1 / 2,1,1)$. The phase portraits of the unperturbed systems are drawn in the two left pictures in Figure 2. In the same way, we can also obtain the 13 small limit cycles bifurcating from the centers of families $\mathcal{T}_{9}$ and $\mathcal{T}_{15}$, fixing now the parameter values to $(\sqrt{5} / 2,3 / 2,-9 / 2,3)$ and $(1,3 \sqrt{7} / 7,-13 \sqrt{7} / 14, \sqrt{7} / 2)$, respectively. The configuration of limit cycles is now $\langle 3,7,3\rangle$. The respective phase portraits are drawn in the two right pictures in Figure 2.

First we take a perturbation of system (5), for the fixed values in $\mathcal{T}_{4}$ mentioned above, keeping the reversible symmetry at the origin,

$$
\begin{aligned}
& \dot{x}=-y-\frac{1}{2} x y+\frac{5}{16} y^{3}+\frac{3}{16} x y^{3}+\sum_{k+2 \ell+1=1}^{4} f_{k, 2 \ell+1} x^{k} y^{2 \ell+1}, \\
& \dot{y}=x+\frac{1}{4} y^{2}-\frac{3}{8} x y^{2}-\frac{3}{32} y^{4}+\sum_{k+2 \ell=1}^{4} f_{k, 2 \ell} x^{k} y^{2 \ell} .
\end{aligned}
$$

It is important to mention that it is not restrictive to assume that the origin remains as an equilibrium point of center type. Then, we do a translation in (13) in order that the center $(-1,2)$ moves to the origin. Moreover, after the translation, the perturbation should start with terms of degree 2 . Because the last limit cycle appears moving the value of the trace at $(-1,2)$ in a usual Hopf bifurcation. This fact restrict the values of the perturbation parameters $f_{k, \ell}$. Now, we can compute and check that the linear developments of the first four Lyapunov quantities are linearly independent. Hence, we have found at least four small limit cycles surrounding $(-1,2)$ and $(-1,-2)$, respectively.

Second, it is enough to consider only a perturbation that does not respect the symmetry around the $x$-axis,

$$
\begin{aligned}
& \dot{x}=-y-\frac{1}{2} x y+\frac{5}{16} y^{3}+\frac{3}{16} x y^{3}+\sum_{k+2 \ell=2}^{4} e_{k, 2 \ell} x^{k} y^{2 \ell}, \\
& \dot{y}=x+\frac{1}{4} y^{2}-\frac{3}{8} x y^{2}-\frac{3}{32} y^{4}+\sum_{k+2 \ell+1=2}^{4} e_{k, 2 \ell+1} x^{k} y^{2 \ell+1} .
\end{aligned}
$$


Here we compute and check, as above, that the linear developments of the first five Lyapunov quantities are linearly independent. Then, we get five small limit cycles surrounding the origin, via a degenerated Hopf bifurcation. We remark that the last limit cycle also appear moving the trace at the origin from zero to a nonzero value, in a usual Hopf bifurcation.

We remark that the parameters that control both bifurcations, (13) and (14), are independent. Considering all together we have at least 13 limit cycles in configuration $\langle 4,5,4\rangle$.

We remark that, in the last result, we have not obtained more limit cycles from a first order analysis because using more Lyapunov quantities we do not have a higher rank to increase the number of limit cycles. Moreover, we have only stated the highest value for the total cyclicity found, studying all the cases listed in Theorem 1.2. We have not done a complete study and we have performed only up to a first order analysis. We have picked a point $(a, b, c, d)$ in each of the 18 families and we have obtained which are the ones that provide the highest cyclicity value. The cases with 13 limit cycles, in configuration $\langle 4,5,4\rangle$, corresponds to families $\mathcal{T}_{4}, \mathcal{T}_{7}, \mathcal{T}_{11}$, and $\mathcal{T}_{13}$ and, in configuration $\langle 3,7,3\rangle$, to families $\mathcal{T}_{1}, \mathcal{T}_{2}, \mathcal{T}_{6}, \mathcal{T}_{9}, \mathcal{T}_{15}$, and $\mathcal{T}_{18}$. Other families provide less limit cycles. The families $\mathcal{T}_{5}, \mathcal{T}_{10}$, and $\mathcal{T}_{16}$ exhibit 11 limit cycles in configuration $\langle 2,7,2\rangle$. Also 11 limit cycles appear in configuration $\langle 3,5,3\rangle$ for families $\mathcal{T}_{8}, \mathcal{T}_{14}$, and $\mathcal{T}_{17}$. Finally, family $\mathcal{T}_{12}$ is the one with less limit cycles, only 9 in configuration $\langle 2,5,2\rangle$.
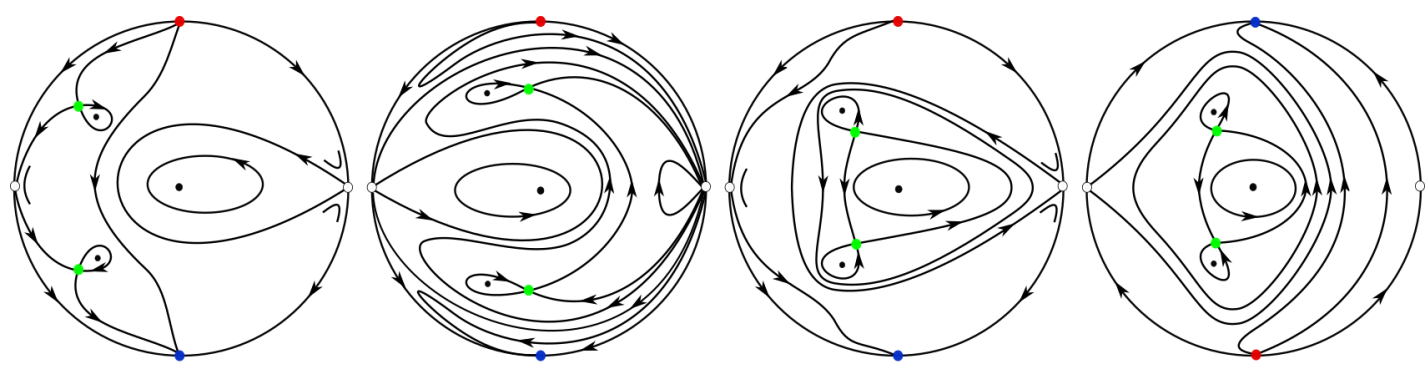

Figure 2. Different phase portraits in the Poincaré disc of system (5)

The equilibrium points in Figure 2 are drawn following the local phase portraits: The centers points in black, the stable nodes in blue, the unstable nodes in red, the saddles in green and the degenerated ones in white. In these last equilibrium points some blowups have been necessary to be done to get the local phase portrait. We remark that the two left centers in Figure 2 have three period annulus while the two right ones have four.

\section{ACKNOWLEDGEMENTS}

This work has been realized thanks to the Brazilian CAPES Agency (grant BEX 13473/13-1), the Catalan AGAUR Agency (grant 2017 SGR 1617), the Slovenian Research Agency (program P1-0306, project N1-0063), and the Spanish Ministerio de Ciéncia, Innovación y Universidades (FEDER grant MTM2016-77278-P).

\section{REFERENCES}

[1] Y. L. Bondar and A. P. Sadovskii. Solution of the center and focus problem for a cubic system that reduces to the Liénard system. Differ. Uravn., 42(1):11-22, 141, 2006.

[2] J. Chavarriga and J. Giné. Integrability of a linear center perturbed by homogeneous polynomial. In Proceedings of the 2nd Catalan Days on Applied Mathematics (Odeillo, 1995), Collect. Études, pages 61-75. Presses Univ. Perpignan, Perpignan, 1995. 
[3] J. Chavarriga and J. Giné. Integrability of cubic systems with degenerate infinity. In XIV CEDYA/IV Congress of Applied Mathematics (Spanish)(Vic, 1995), page 12. Univ. Barcelona, Barcelona, 1996.

[4] C. Chicone and M. Jacobs. Bifurcation of critical periods for plane vector fields. Trans. Amer. Math. Soc., 312(2):433-486, 1989.

[5] C. Chicone and M. Jacobs. Bifurcation of limit cycles from quadratic isochrones. J. Differential Equations, 91(2):268-326, 1991.

[6] C. Christopher. Estimating limit cycle bifurcations from centers. In Differential equations with symbolic computation, Trends Math., pages 23-35. Birkhäuser, Basel, 2005.

[7] D. Cozma. The problem of the center for cubic systems with two parallel invariant straight lines and one invariant cubic. ROMAI J., 11(2):63-75, 2015.

[8] D. Cozma and A. Şubă. The solution of the problem of center for cubic differential systems with four invariant straight lines. An. Ştiinţ. Univ. Al. I. Cuza Iaşi. Mat. (N.S.), 44(suppl.):517-530 (2000), 1998. Mathematical analysis and applications (Iaşi, 1997).

[9] W. Decker, G.-M. Greuel, G. Pfister, and H. Schönemann. Singular 4-1-1 - A computer algebra system for polynomial computations. http://www.singular.uni-kl.de, 2018.

[10] W. Decker, G. Pfister, H. Schönemann, and S. Laplagne. primdec.lib a Singular 4-1-1 library for computing the primary decomposition and radical ideals, 2018.

[11] H. Dulac. Détermination et intégration d'une certaine class d'équations différentialles ayant pour point singulier un centre. Bull. Sci. Math., 32(2):230-252, 1908.

[12] F. Dumortier, J. Llibre, and J. C. Artés. Qualitative theory of planar differential systems. Universitext. Springer-Verlag, Berlin, 2006.

[13] B. Ferčec, J. Giné, V. G. Romanovski, and V. F. Edneral. Integrability of complex planar systems with homogeneous nonlinearities. J. Math. Anal. Appl., 434(1):894-914, 2016.

[14] M. Frommer. über das Auftreten von Wirbeln und Strudeln (geschlossener und spiraliger Integralkurven) in der Umgebung rationaler Unbestimmtheitsstellen. Math. Ann., 109(1):395-424, 1934.

[15] P. Gianni, B. Trager, and G. Zacharias. Gröbner bases and primary decomposition of polynomial ideals. J. Symbolic Comput., 6(2-3):149-167, 1988. Computational aspects of commutative algebra.

[16] M. Han. Liapunov constants and Hopf cyclicity of Liénard systems. Ann. Differential Equations, 15(2):113-126, 1999.

[17] Y. Ilyashenko and S. Yakovenko. Lectures on analytic differential equations, volume 86 of Graduate Studies in Mathematics. American Mathematical Society, Providence, RI, 2008.

[18] W. Kaptyen. On the midpoints of integral curves of differntial equations of the first degree. Nederl. Adak. Wetecnsch. Verslag. Afd. Natuurk. Konikl., pages 1446-1457, 1911.

[19] W. Kaptyen. New investigations on the midpoints of integral of differential equations of the first degree. Nederl. Adak. Wetecnsch. Verslag. Afd. Natuurk. Konikl., 20:1354-1365, 1912.

[20] A. M. Liapunov. Investigation of one of the special cases of the stability of motion. Mat. Sb., 17:253$333,1893$.

[21] A. M. Liapunov. Problème Général de la Stabilité du Mouvement. Annals of Mathematics Studies, no. 17. Princeton University Press, Princeton, N. J.; Oxford University Press, London, 1947.

[22] N. G. Lloyd, C. J. Christopher, J. Devlin, J. M. Pearson, and N. Yasmin. Quadratic-like cubic systems. Differential Equations Dynam. Systems, 5(3-4):329-345, 1997. Planar nonlinear dynamical systems (Delft, 1995).

[23] H. Poincaré. Mémoire sur les courbes définies par une équation différentielle. Sér.3)7(1881), 375-422; Sér.(3)8(1882),251-296; Sér.(4)1(1885),167-244; Sér. (4)2(1886), 151-217.

[24] R. Prohens and J. Torregrosa. New lower bounds for the Hilbert numbers using reversible centers. Nonlinearity, 32(331), 2019.

[25] V. G. Romanovski and D. S. Shafer. The center and cyclicity problems: a computational algebra approach. Birkhäuser Boston, Inc., Boston, MA, 2009.

[26] V. G. Romanovski and D. S. Shafer. Centers and limit cycles in polynomial systems of ordinary differential equations. In School on real and complex singularities in São Carlos, 2012, volume 68 of Adv. Stud. Pure Math., pages 267-373. Math. Soc. Japan, [Tokyo], 2016.

[27] R. Roussarie. Bifurcation of planar vector fields and Hilbert's sixteenth problem, volume 164 of Progress in Mathematics. Birkhäuser Verlag, Basel, 1998.

[28] N. A. Saharnikov. On Frommer's conditions for the existence of a center. Akad. Nauk SSSR. Prikl. Mat. Meh., 12:669-670, 1948.

[29] B. Sang. Center-focus problems for two classes of cubic differential systems. J. Nanjing Norm. Univ. Nat. Sci. Ed., 35(2):16-21, 2012. 
[30] B. Sang and C. Niu. Solution of center-focus problem for a class of cubic systems. Chin. Ann. Math. Ser. B, 37(1):149-160, 2016.

[31] K. S. Sibirskiǐ. On conditions for the presence of a center and a focus. Kišinev. Gos. Univ. Uč. Zap., 11:115-117, 1954.

[32] K. S. Sibirskir. The principle of symmetry and the problem of the center. Kišinev. Gos. Univ. Uč. Zap., 17:27-34, 1955.

[33] K. S. Sibirski1. On the number of limit cycles in the neighborhood of a singular point. Differencialnye Uravnenija, 1:53-66, 1965.

[34] P. S. Wang, M. J. T. Guy, and J. H. Davenport. P-adic reconstruction of rational numbers. SIGSAM Bull., 16(2):2-3, 1982.

[35] H. Żoładek. Quadratic systems with center and their perturbations. J. Differential Equations, 109(2):223-273, 1994.

Departament de Matemàtiques, Universitat Autònoma de Barcelona, 08193 Bellaterra, Barcelona, Catalonia, Spain

Email address: leonardo@mat.uab.cat

Faculty of Electrical Engineering and Computer Science, University of Maribor, KorošKa cesta 46, SI-2000, Maribor, Slovenia

Center for Applied Mathematics and Theoretical Physics, University of Maribor, Mladinska 3, SI-2000, Maribor, Slovenia

Faculty of Natural Science and Mathematics, University of Maribor, Koroška cesta 160, Si-2000, Maribor, Slovenia

Email address: valerij.romanovskij@um.si

Departament de Matemàtiques, Universitat Autònoma de Barcelona, 08193 Bellaterra, Barcelona, Catalonia, Spain

Email address: torre@mat.uab.cat 